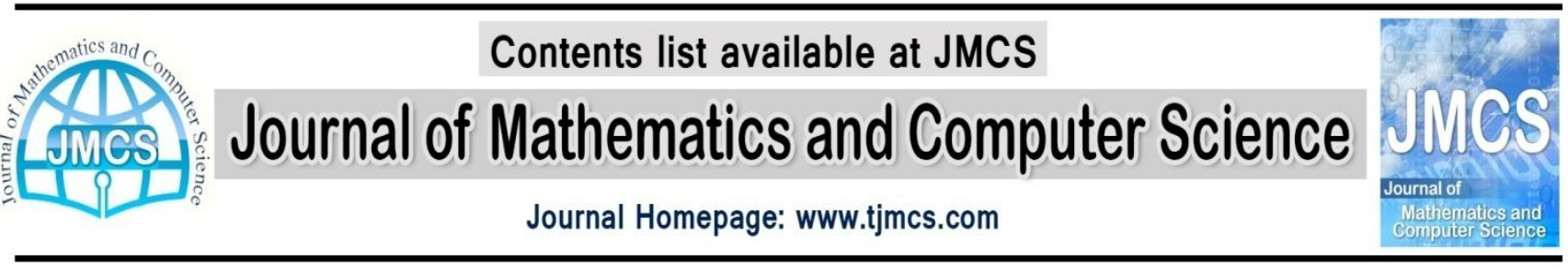

\title{
Variational Homotopy Perturbation Method: An efficient scheme for solving partial differential equations in fluid mechanics
}

\author{
T. Allahviranloo ${ }^{1}$ A. Armand ${ }^{2, *}$, S. Pirmuhammadi ${ }^{1}$ \\ ${ }^{1}$ Department of Mathematics, Science and Research Branch, Islamic Azad University, Tehran, Iran \\ ${ }^{2}$ Young Researchers and Elite Club, Shahr-e-Rey Branch, Islamic Azad University, Tehran, Iran \\ tofigh@allahviranloo.com, atefeh.armand@ymail.com, s.pirmuhammadi@hotmail.com
}

\section{Article history:}

Received : August 2013

Accepted : September 2013

Available online : January 2014

\begin{abstract}
In this paper, an application of variationalhomotopy perturbation method is applied to solve Korteweg-de Vries (KdV)and Burgers equations. The study reveals that the method is very effective and simple.
\end{abstract}

Keywords: Variational homotopy perturbation method, Korteweg-de Vries equation, Burgers equation.

\section{Introduction}

It is well known that there are many phenomena in engineering, biology, fluid mechanics and other sciences can be modeled as partial differential equations (PDEs), such as KdV equation, Burgers equation, Fisher's equation, wave equation, biharmonic equation and many other important equations. These equations are usually difficult to solve analytically, so these are required to obtain efficient approximately solutions. Therefore, in recent years many authors have studied on solutions of PDEs by some numerical methods. For example, the Adomian decomposition method (ADM) was employed in [8] for solving generalized Boussinesq equation. Application of the variational iteration method (VIM) to the $\mathrm{KdV}, \mathrm{k}(2,2)$, Burgers and cubic Boussinesq equations are investigated in [22]. He's variational iteration technique is used in [2] for solving Klein-Gordon equation. In [3, 23], the applications of the VIM to burgers, coupled Burgers equation and Blasius equation are provided. The homotopy perturbation method (HPM) is used in [7] for solving KdV type equations. The authors of [20] applied the homotopy analysis method (HAM) to the KdV equation.

In this paper, we extend the application of the variational homotopy perturbation method (VHPM) to find approximate solutions for the $\mathrm{KdV}$ and Burgers equations. The VHPM which proposed in [16, 14], is based on the HPM and the VIM. The VIM is a simple and effective method which proposed by Chinese

\footnotetext{
${ }^{*}$ Corresponding author.
} 
mathematician Ji-Huan He [9-12] as modification of a general Lagrange multiplier method [13]. The homotopy perturbation method $[4,15,17]$ has been used by many mathematicians and engineers to solve various functional equations. The HPM, using the homotopy technique of topology, a homotopy is constructed with an embedding parameter $p \in[0,1]$ which considered as a small parameter. This method, which does not require a small parameter, which is the case with other methods, has a significant advantage in that it provides an approximate solution to a wide range of nonlinear problems in applied science [21]. Recently, the application of the variational homotopy perturbation method has been extended to higher dimensional initial boundary problems [16]. This technique is used in [14] for solving the Fisher's equation. Moreover, the VHPM was successfully applied to nonlinear oscillators [6]. For a relatively comprehensive survey of the methods and their applications, the reader is referred to [16].

Consider the $\mathrm{KdV}$ equation,

$$
u_{t}-3\left(u^{2}\right)_{x}+u_{3 x}=0
$$

First derived by Korteweg de Vries in their study of long water waves in a (relatively shallow) channel in 1895. Also, Eq. (1.1) is the pioneering equation that gives rise to solitary wave solutions. Solutions which are waves with infinite support are generated as a result of the balance between the nonlinear convection $\left(u^{n}\right) x$ and the linear dispersion $u_{3 x}$ in these equations. Solutions are localized waves that propagate without change of their shape and velocity properties and stable against mutual collisions [22].

The Burgers equation

$$
u_{t}+\frac{1}{2}\left(u^{2}\right)_{x}-u_{2 x}=0
$$

appears in fluid mechanics. This equation incorporates both convection and diffusion in fluid dynamics, and is used to describe the structure of shock waves [22]. In the above equations, the unknown function $u=u(x, t)$ is sufficiently often differentiable function and it is usually assumed to be real.

Subscriptsdenote partial differentiations, that is $u_{k x}=\partial^{k} u / \partial x^{k}, k=1,2,3$ and $u_{t}=\partial u / \partial t$ for $x \in \square, t>0$.

The following structure leads this paper as follows: In section 2, we introduce the variational homotopy perturbation method. In section 3, we try to implement the VHPM on the KdV and Burgers equations. In the last section, we will present the results of this work.

\section{The Method}

In this section, we will highlight briefly the main point of the variational homotopy perturbation method (VHPM), where details can be found in $[6,14,16]$.

To clarify the VHPM, let us assume the following nonlinear differential equation the form

$$
L(u(x, t))+N(u(x, t))=g(t),
$$

where $L$ and $N$ are linear and nonlinear operators, respectively and $g(t)$ is a known analytical function. According to the variational iteration method, we write down a correction functional

$$
u_{n+1}(x, t)=u_{n}(x, t)+\int_{0}^{x} \lambda(x, s)\left[L u_{n}(x, s)+N \tilde{u}_{n}(x, s)-g(s)\right] d s,
$$

where $\lambda$ is a general Lagrangian multiplier, which can be determined optimally via the variational theory, $u_{n}$ is the $n^{\text {th }}$ approximate solution and $\tilde{u}_{n}$ denotes a restricted variation, i.e. $\delta \tilde{u}_{n}=0$.

Now, by homotopy perturbation method $[1,5]$, we can construct an equation as follows

$$
\sum_{i=0}^{\infty} p^{i} u_{i}=u_{0}(x)+p \int_{0}^{x} \lambda(x, s)\left[\sum_{i=0}^{\infty} p^{i} L\left(u_{i}(x, s)\right)+N\left(\sum_{i=0}^{\infty} p^{i} \tilde{u}_{i}(x, s)\right)\right] d s-\int_{0}^{x} \lambda(x, s) g(s) d s .
$$


T. Allahviranloo, A. Armand, S. Pirmuhammadi/ J. Math. Computer Sci. 9 (2014), 362-369

By equating the terms (2.3) with identical powers of $p$, and taking the limit as $p$ tends to 1 , we get

$$
u(x, t)=\lim _{p \rightarrow 1} \sum_{i=0}^{\infty} p^{i} u_{i}(x, t)=u_{0}(x, t)+u_{1}(x, t)+u_{2}(x, t)+\cdots
$$

\section{The Governing Equations}

In what follows, we will apply the variational homotopy perturbation method to the $\mathrm{KdV}$ and Burgers equations.

\subsection{The KdV equation}

First, we consider Eq.(1.1) as

$$
u_{t}-3\left(u^{2}\right)_{x}+u_{3 x}=0, \quad-\infty<x<+\infty, \quad t>0,
$$

with initial condition

$$
u(0, x)=6 x
$$

where subscripts denote differentiations.

By means of the VHPM, we consider

$$
L(u)=u_{t},
$$

and

$$
N(u)=-3\left(u^{2}\right)_{x}+u_{3 x}
$$

where $L$ is a linear operator and $N$ is a nonlinear operator. In order to construct a correction functional for this system,we can write down the following expression

$$
u_{n+1}(x, t)=u_{n}(x, t)+\int_{0}^{t} \lambda(x, s)\left[\left(u_{n}(x, s)\right)_{t}-3\left(\tilde{u}_{n}^{2}(x, s)\right)_{x}+\left(\tilde{u}_{n}(x, s)\right)_{3 x}\right]
$$

where $\tilde{u}_{n}$ denote a restricted variation, i.e. $\delta\left(\tilde{u}_{n}\right)=0$. To find the optimal value of $\lambda$, we make the correction functional(3.4) stationary in the following form

$$
\begin{aligned}
\delta u_{n+1}(x, t) & =\delta u_{n}(x, t)+\delta \int_{0}^{t} \lambda(x, s)\left[\left(u_{n}(x, s)\right)_{t}-3\left(\tilde{u}_{n}^{2}(x, s)\right)_{x}+\left(\tilde{u}_{n}(x, s)\right)_{3 x}\right] d s \\
= & \delta u_{n}(x, t)+\left.\lambda(x, s) \delta u_{n}(x, s)\right|_{s=t}-\int_{0}^{t} \frac{\partial \lambda(x, s)}{\partial s} \delta u_{n}(x, s) d s \\
-\int_{0}^{t} \frac{\partial \lambda(x, s)}{\partial s}-3 \delta\left(\tilde{u}_{n}^{2}(x, s)\right)_{x}+\partial\left(\tilde{u}_{n}(x, s)\right)_{3 x} d s & \\
= & \left.(1+\lambda(x, s)) \delta u_{n}(x, s)\right|_{s=t}-\int_{0}^{t} \frac{\partial \lambda(x, s)}{\partial s} \delta u_{n}(x, s) d s=0 .
\end{aligned}
$$

Hence, we have the following stationary conditions 


$$
\begin{aligned}
& \left.\frac{\partial}{\partial s} \lambda(x, s)\right|_{s=t}=0, \\
& 1+\left.\lambda(x, s)\right|_{s=t}=0 .
\end{aligned}
$$

This in turn gives $\lambda(x, s)=-1$.By substituting $\lambda$ into Eq. (3.4), the following formula is obtained

$$
u_{n+1}(x, t)=u_{n}(x, t)-\int_{0}^{t}\left[\left(u_{n}(x, s)\right)_{t}-3\left(u_{n}^{2}(x, s)\right)_{x}+\left(u_{n}(x, s)\right)_{3 x}\right] d s .
$$

Then, Eq. (3.5) will enable us to determine the components $u_{n}(x, t)$ recursively for $n \geq 0$.

Now, by exerting the VHPM, it is then possible to obtain an equation as follows

$$
\begin{gathered}
\boldsymbol{u}_{0}+p \boldsymbol{u}_{1}+p^{2} \boldsymbol{u}_{2}+\cdots=6 x+3 p \int_{0}^{t}\left[\left(\left(u_{0}+p u_{1}+p^{2} u_{2}+\cdots\right)^{2}\right)_{x}\right] d s \\
-p \int_{0}^{t}\left[\left(u_{0}+p u_{1}+p^{2} u_{2}+\cdots\right)_{3 x}\right] d s \\
=6 x+3 p \int_{0}^{t}\left[\left(u_{0}^{2}\right)_{x}+p\left(2 u_{0} u_{1}\right)_{x}+p^{2}\left(u_{1}^{2}+2 u_{0} u_{2}\right)_{x}+\cdots\right] d s \\
-p \int_{0}^{t}\left[\left(u_{0}\right)_{3 x}+p\left(u_{1}\right)_{3 x}+p^{2}\left(u_{2}\right)_{3 x}+\cdots\right] d s .
\end{gathered}
$$

By comparing the terms with identical powers of $p$, we have the following results

$$
\begin{aligned}
& p^{0}: u_{0}(x, t)=6 x, \\
& p^{1}: u_{1}(x, t)=3 \int_{0}^{t}\left(u_{0}^{2}\right)_{x} d s-\int_{0}^{t}\left(u_{0}\right)_{3 x} d s=216 x t, \\
& p^{2}: u_{2}(x, t)=3 \int_{0}^{t}\left(2 u_{0} u_{1}\right)_{x} d s-\int_{0}^{t}\left(u_{1}\right)_{3 x} d s=7776 x t^{2}, \\
& p^{3}: u_{3}(x, t)=3 \int_{0}^{t}\left(u_{1}^{2}+2 u_{0} u_{2}\right)_{x} d s-\int_{0}^{t}\left(u_{2}\right)_{3 x} d s=279936 x t^{3}, \\
& \vdots
\end{aligned}
$$

By calculating seven terms of the series, an approximate solution of the VHPM is obtained

$$
u(x, t) \cong \phi_{7}(x, t)=\lim _{p \rightarrow 1} \sum_{i=0}^{6} p^{i} u_{i}(x, t)=6 x\left(1+36 t+(36 t)^{2}+(36 t)^{3}+(36 t)^{4}+(36 t)^{5}+(36 t)^{6}\right) .
$$

So the solution in a closed form is:

$$
u(x, t)=\frac{6 x}{1-36 t}
$$

If we set $t=0.005$ and $t=0.01$, comparing the VHPM and the VIM with exact solution yields our method is more accurate. Results are shown inTable(3.1)and Fig. (3.1). 
T. Allahviranloo, A. Armand, S. Pirmuhammadi/ J. Math. Computer Sci. 9 (2014), 362-369

Table (3. 1). Absolute error of the KdV equation

\begin{tabular}{|c|c|c|c|c|}
\hline $\boldsymbol{x}$ & VIM in $t=0.005$ & VHPM in $t=0.005$ & VIM in $t=0.01$ & VHPM in $t=0.01$ \\
\hline 0.1 & $3.09037 E-03$ & $4.47966 E-06$ & $3.39311 E-02$ & $7.34664 E-04$ \\
\hline 0.2 & $6.18074 E-03$ & $8.95932 E-06$ & $6.78622 E-02$ & $146933 E-03$ \\
\hline 0.3 & $9.27112 E-03$ & $1.34390 E-05$ & $1.01793 E-01$ & $220399 E-03$ \\
\hline 0.4 & $1.23615 E-02$ & $1.79186 E-05$ & $1.35724 E-01$ & $293866 E-03$ \\
\hline 0.5 & $1.54519 E-02$ & $2.23983 E-05$ & $1.69655 E-01$ & $367332 E-03$ \\
\hline 0.6 & $1.85422 E-02$ & $2.68780 E-05$ & $2.03587 E-01$ & $440798 E-03$ \\
\hline 0.7 & $2.16326 E-02$ & $3.13576 E-05$ & $2.37518 E-01$ & $514265 E-03$ \\
\hline 0.8 & $2.47230 E-02$ & $3.58373 E-05$ & $2.71449 E-01$ & $587731 E-03$ \\
\hline 0.9 & $2.78134 E-02$ & $4.03169 E-05$ & $3.05380 E-01$ & $661198 E-03$ \\
\hline 1.0 & $3.09037 E-02$ & $4.47966 E-05$ & $3.39311 E-01$ & $734664 E-03$ \\
\hline
\end{tabular}

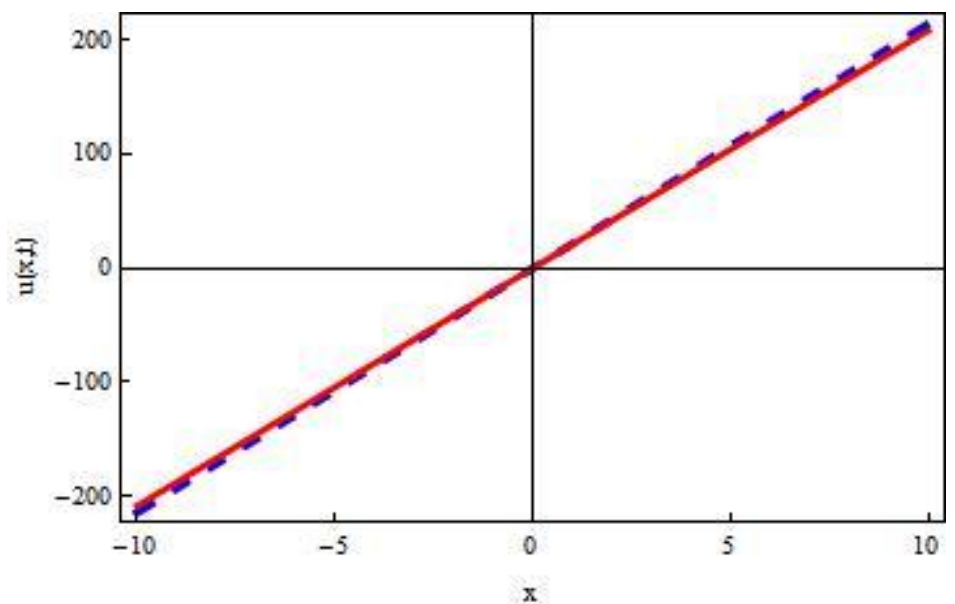

Fig. (3. 1). Comparison of the exact solution with approximate solution (3. 6) of the KdV equation for $t=1$ and $-10 \leq x \leq 10$ .Symbols: solid line: exact solution; dash line: VHPM

\subsection{The Burgers equation}

Our last consideration is to takeplacein Burgers equation as

$$
u_{t}+\frac{1}{2}\left(u^{2}\right)_{x}-u_{2 x}=0, \quad t>0, \quad x \in \square
$$

with the following initial condition

$$
u(x, 0)=x
$$

By means of the first step of the VHPM, we assume that

$$
L(u)=u_{t},(\mathbf{3 . 8})
$$

and 


$$
N(u)=\frac{1}{2}\left(u^{2}\right)_{x}-u_{2 x} .
$$

Now, like before we try to construct the correction functional. So, take the form of

$$
u_{n+1}(x, t)=u_{n}(x, t)+\int_{0}^{t} \lambda(x, s)\left[\left(u_{n}(x, s)\right)_{t}+\frac{1}{2}\left(\tilde{u}_{n}^{2}(x, s)\right)_{x}-\left(\tilde{u}_{n}(x, s)\right)_{2 x}\right] d s,
$$

again we obtain $\lambda(x, s)=-1$. Therefore, Eq. (3.10) changes to

$$
u_{n+1}(x, t)=u_{n}(x, t)-\int_{0}^{t}\left[\left(u_{n}(x, s)\right)_{t}+\frac{1}{2}\left(u_{n}^{2}(x, s)\right)_{x}-\left(u_{n}(x, s)\right)_{2 x}\right] d s .
$$

Now, by preforming the VHPM, it is then possible to obtain the following relation

$$
\begin{gathered}
u_{0}+p u_{1}+p^{2} u_{2}+\cdots=x-\frac{1}{2} p \int_{0}^{t}\left[\left(\left(u_{0}+p u_{1}+p^{2} u_{2}+\cdots\right)^{2}\right)_{x}\right] d s \\
+p \int_{0}^{t}\left[\left(u_{0}+p u_{1}+p^{2} u_{2}+\cdots\right)_{2 x}\right] d s \\
=x-\frac{1}{2} p \int_{0}^{t}\left[\left(u_{0}^{2}\right)_{x}+p\left(2 u_{0} u_{1}\right)_{x}+p^{2}\left(u_{1}^{2}+2 u_{0} u_{2}\right)_{x}+\cdots\right] d s \\
+p \int_{0}^{t}\left[\left(u_{0}\right)_{2 x}+p\left(u_{1}\right)_{2 x}+p^{2}\left(u_{2}\right)_{2 x}+\cdots\right] d s .
\end{gathered}
$$

By equating the coefficients of $p$ with the same power, one gets

$$
\begin{aligned}
& p^{0}: u_{0}(x, t)=x, \\
& p^{1}: u_{1}(x, t)=-\frac{1}{2} \int_{0}^{t}\left(u_{0}^{2}\right)_{x} d s+\int_{0}^{t}\left(u_{0}\right)_{2 x} d s=-x t, \\
& p^{2}: u_{2}(x, t)=-\frac{1}{2} \int_{0}^{t}\left(2 u_{0} u_{1}\right)_{x} d s+\int_{0}^{t}\left(u_{1}\right)_{2 x} d s=x t^{2}, \\
& p^{3}: u_{3}(x, t)=-\frac{1}{2} \int_{0}^{t}\left(u_{1}^{2}+2 u_{0} u_{2}\right)_{x} d s+\int_{0}^{t}\left(u_{2}\right)_{2 x} d s=-x t^{3}, \\
& \vdots
\end{aligned}
$$

By calculating seven terms of the series, an approximate solution of the VHPM is obtained

$$
u(x, t) \cong \phi_{7}(x, t)=\lim _{p \rightarrow 1} \sum_{i=0}^{6} p^{i} u_{i}(x, t)=x\left(1-t+(t)^{2}-(t)^{3}+(t)^{4}-(t)^{5}+(t)^{6}\right),
$$

with the following closed form

$$
u(x, t)=\frac{x}{1+t} .
$$


T. Allahviranloo, A. Armand, S. Pirmuhammadi/ J. Math. Computer Sci. 9 (2014), 362-369

The results corresponding absolute errors are presented in Table (3.2)and Fig. (3.2).

Table (3.2). Absolute error of the Burgers equation

\begin{tabular}{|c|c|c|c|}
\hline $\boldsymbol{x}$ & $\mathbf{0 . 0 0 5}$ & $\mathbf{0 . 0 1}$ & $\boldsymbol{0 . 1}$ \\
\hline 0.1 & $1.38777 E-17$ & $9.85323 E-16$ & $9.09091 E-09$ \\
0.2 & $2.77555 E-17$ & $1.97065 E-15$ & $1.81818 E-08$ \\
0.3 & $5.55111 E-17$ & $2.94209 E-15$ & $2.72727 E-08$ \\
0.4 & $5.55111 E-17$ & $3.94129 E-15$ & $3.63636 E-08$ \\
0.5 & $5.55111 E-17$ & $4.94049 E-15$ & $4.54545 E-08$ \\
0.6 & $1.11022 E-16$ & $5.88418 E-15$ & $5.45455 E-08$ \\
0.7 & $1.11022 E-16$ & $6.88338 E-15$ & $6.36364 E-08$ \\
0.8 & $1.11022 E-16$ & $7.88258 E-15$ & $7.27273 E-08$ \\
0.9 & $1.11022 E-16$ & $8.88178 E-15$ & $8.18182 E-08$ \\
1.0 & $1.11022 E-16$ & $9.88098 E-15$ & $9.09091 E-08$ \\
\hline
\end{tabular}

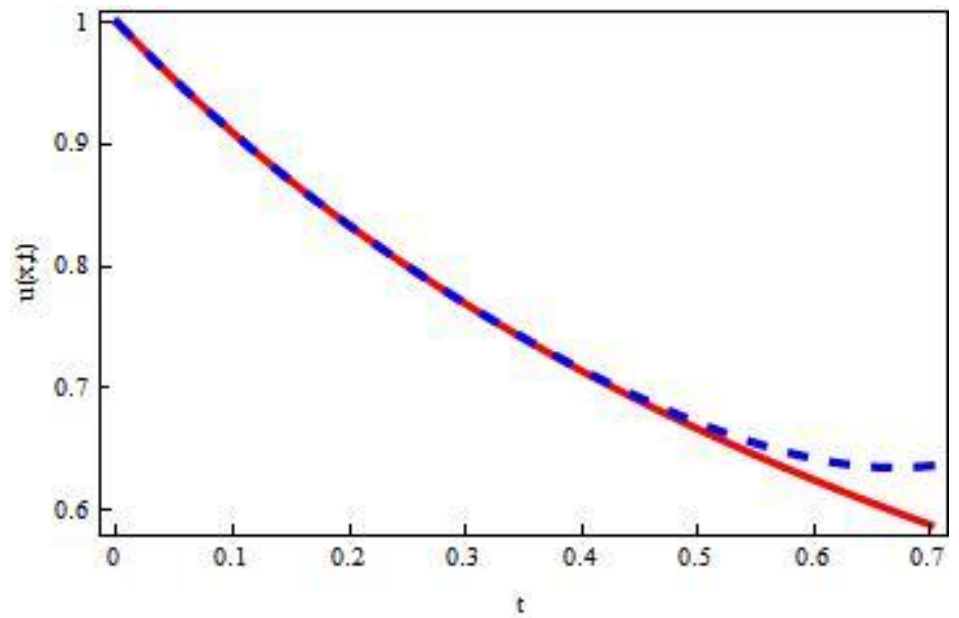

Fig. (3.2). Comparison of the exact solution with approximate solution (3.12) of the Burgers equation for $x=1$ and $0 \leq t \leq 0.7$ .Symbols: solid line: exact solution; dash line: VHPM

\section{Conclusion}

In this paper, the variational homotopy perturbation method was successfully employed for solving the $\mathrm{KdV}$ and Burgers equations. This method is based onthe homotopy perturbation method and variational iteration method. For our equations, the results of this method are exactly the same as those obtained bythe homotopy perturbation method. As an advantage of this method over the homotopy perturbation method, we do not need to solve a differential equation in eachiteration. It is important to note that this technique unlike most numerical method provides a closed form of the solution. The computations in this paper were performed by using Mathematica 7. 


\section{References}

[1] S. Abbasbandy, Application of He's homotopy perturbation method for Laplace transform, Chaos, Solitons and Fractals 30 (5) (2006) 1206-1212.

[2] S. Abbasbandy, Numerical solutions of nonlinear Klein-Gordon equation by variational iteration method. Internat. J. Numer. Meth. Engrg. 70 (2007) 876-881.

[3] M.A. Abdou, A.A. Soliman, variational iteration method for solving Burger's and coupled Burger's equations,J. Compu. Appl. Math. 181(2005) 245-251.

[4] M. Bagheri, M. Bagheri, E. Miralikatouli, Comparison Differential Transform Method with Homotopy Perturbation Method for Nonlinear Integral Equations, Journal of mathematics and computer Science 5 (2012) 288-296.

[5] L. Cveticanin, Homotopy-perturbation method for pure nonlinear differential equation, Chaos, Solitons and Fractals 30 (5) (2006) 1221-1230.

[6] F. M. Fernandez, On the variational homotopy perturbation method for nonlinear oscillators, Mathematical physics 1 (2011) $1-3$.

[7] H. Goodarzian, T. Armaghani and M. Okazi, Study of nonlinear KdV type equations via homotopy perturbation method and variational iteration method, International Journal of the Physical Sciences 7(2) (2012) 234 - 240.

[8] M.A. Hajji, K. Al-Khaled, Analytic studies and numerical simulations of the generalized Boussinesq equation, Applied Mathematics and Computation 191 (2007) 320-333.

[9] J.H. He, Variational iteration method -some recent results and new interpretations-, J. Comput. Appl. Math. 207 (1) (2007) 317.

[10] J.H. He, Variational principles for some nonlinear partial differential equations with variable coefficients, Chaos, Solitons and Fractals 19 (2004) 847-851.

[11] J.H. He, Variational iteration method for autonomous ordinary differential systems, Appl. Math. Comput. 114 (2000) 115123.

[12] J.H. He, Some asymptotic methods for strongly equations, Int. J. Mod. Phys. B 20 (10) (2006) 1141-1199.

[13] M. Inokuti, General use of the Lagrange multiplier in non-linear mathematical physics, in: S. Nemat-Nasser (Ed.), Variational Method in the Mechanics of Solids, Pergamon Press, Oxford (1978) 156-162.

[14] M. Matinfar, M. Mahdavi, Z. Raeisy, The implementation of variational homotopy perturbation method for Fisher's equation, International journal of nonlinear science 9 (2010) 188-194.

[15] M. Mahmoudi, M.V. Kazemi, Solving Singular BVPs Ordinary Differential Equations by Modified Homotopy Perturbation Method, Journal of mathematics and computer Science 7 (2013) 138 - 143.

[16] M.A. Noor, S.T. Mohyud-Din, Variational homotopy perturbation method for solving higher dimensional initial boundary problems, Mathematical problems in engineering (2008) 1-11.

[17] M. Rabbani, New Homotopy Perturbation Method to Solve Non-Linear Problems, Journal of mathematics and computer Science 7 (2013) 272 - 275.

[18] M. Rabbani, R. Jamali, Solving Nonlinear System of Mixed Volterra-Fredholm Integral Equations by Using Variational Iteration Method 5 (2012) 280-287.

[19] P. Rosenau, J.M. Hyman, Compactons: solitons with finite wavelengths, Phys. Rev. Lett. 70 (5) (1993) $564-567$.

[20] F. Saadi, M. Jalali Azizpour, S.A. Zahedi, Analytical Solutions of Kortweg-de Vries (KdV) Equation, World Academy of Science, Engineering and Technology 70 (2010) 171-175.

[21] E. Yusufo glu, Improved homotopy perturbation method for solving Fredholm type integro-differential equations, Chaos, Solitons and Fractals 41 (2009) 28-37.

[22] A.M. Wazwaz, The variational iteration method for rational solutions for $\mathrm{KdV}, \mathrm{K}(2,2)$, Burgers, and cubic Boussinesq equations, Journal of Computational and Applied Mathematics 207 (1) (2007) 18-23.

[23] A.M. Wazwaz, The volitionaliteration method for solving two forms of Blasius equation on a half-infinite domain, Appl. Math. Comput. 114 (2006) 115-123. 